wissen kompakt 2011 · 5:2

DOI 10.1007/s11838-011-0123-6

Online publiziert: 24. Juli 2011

(c) Springer-Verlag und Freier Verband Deutscher

Zahnärzte e.V. 2011

\section{N. Grosse}

Wiesbaden

\section{Zahnerhaltung}

\section{Zusammenspiel von Wissenschaft, Praxis und industriellen Entwicklungen}

Würde man die ethische Messlatte an alle zahnmedizinischen Leistungen legen, so stünden die präventiven und zahnerhaltenden Maßnahmen sicher weit oben auf der Skala. Ohne die schönsten prothetischen Rekonstruktionen oder das perfekte Implantat mindern zu wollen, die Zahnerhaltung ist und bleibt das zentrale, gemeinsame Anliegen von Zahnarzt und Patient in der täglichen Praxis. Nach wie vor gilt das Ausmaß des Zahnverlusts als wichtiges Kriterium für die Zahngesundheit.

\section{(2) Ein Risikofaktor für Zahnverlust ist der zu seltene Zahnarztbesuch}

Dass es ohne Zweifel Erfolge auf dem Weg dieser Bemühungen gibt, zeigen die seit 1989 durchgeführten Mundgesundheitsstudien. In der kürzlich vom Institut der Deutschen Zahnärzte (IDZ) veröffentlichten Studie über die Ursachen von Zahnverlust in Deutschland (Glockmann et al.) werden die „beachtlichen epidemiologischen Veränderungen für die Prävalenz der Karies und Parodontitiden und in der Folge den Zahnverlust" aufgezeigt: Insgesamt zeigt sich ein Rückgang der Karies, die Prävalenz parodontaler Erkrankungen steigt mit zunehmenden Alter unserer Patienten an. Entsprechend wurden nach der Untersuchung von Glockmann et al. in Deutschland bleibende Zähne zahlenmäßig häufiger aufgrund des Vorliegens einer Parodontitis extrahiert, während „bei Berücksichtigung aller Zähne (inkl. der Milchzähne) die Ka- ries geringfügig öfter eine Zahnentfernung verursachte“.

Als Risikofaktor für Zahnverlust wird dabei u. a. der zu seltene Zahnarztbesuch genannt. Somit kommt der frühzeitigen Diagnose der Zahnkaries bei den Aufgaben des Zahnarztes ein besonderer Stellenwert zu. Dass der Zahnarzt dabei neben Spiegel, Sonde und Röntgenbild auf neue unterstützende technische Verfahren zurückgreifen kann, zeigt der Beitrag aus der Marburger Klinik zu den aktuellen Entwicklungen in der Kariesdiagnostik.

Zunehmend sind es aber auch die nichtkariösen Zahnhartsubstanzdefekte, die in der Zahnarztpraxis beobachtet werden. Die infolge chemischer Erosion und/oder mechanischer Abrasion hervorgerufenen ästhetischen und funktionellen Störungen stellen den Zahnarzt vor große Herausforderungen. Eine restaurative, nichtinvasive Möglichkeit der Restauration wird von der Züricher Klinik vorgestellt.

Voraussetzung für solche umfangreichen Restaurationen ist ein funktionierender Haftungsmechanismus der Restaurationen an Zahnschmelz und Dentin, den uns die mikromechanische Verankerung durch die Ädhäsivsysteme gebracht hat - ohne Frage eine der größten Entwicklung im Bereich der Zahnerhaltung der letzten Jahrzehnte. Der Beitrag von U. Blunck greift die besondere Fragestellung auf, ob die selbstätzenden Ädhäsivsysteme die techniksensible Phosphorsäureätzung von Schmelz und Dentin schon ersetzen können.
Eine schöne und besondere Variante einer effektiven Zahnerhaltungsmaßnahme ohne aufwendige Restaurationen zeigt das Fallbeispiel aus der Baseler Klinik zum Einsatz der externen Bleichtherapie nach Zahntrauma und Zahnverfärbung.

Alle vier Beiträge sind Beweis dafür, wie das Zusammenspiel von Wissenschaft, Praxis und industriellen Entwicklungen auch in kleinen und vielleicht weniger spektakulären Schritten zur Zahnerhaltung unserer Patienten und zur Verbreitung weniger invasiver Methoden beitragen können.

Ich bedanke mich bei allen Autoren für ihre Beiträge und hoffe, dass diese auch neue Informationen für die tägliche Arbeit in der Praxis bringen können.

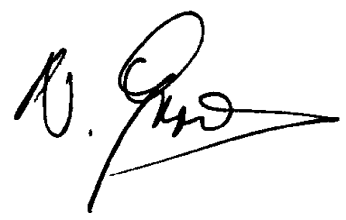

N. Grosse

Korrespondenzadresse Dr. N. Grosse

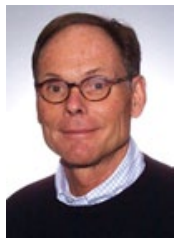

Theodorenstr. 5, 64189 Wiesbaden n.grosse@t-online.de 